Frank A. Orlando, MD; Ku-Lang Chang, MD; Irene M. Estores, MD Department of Community Health and Family Medicine (Drs. Orlando and Chang) and Department of Physical Medicine and Rehabilitation (Dr. Estores), University of Florida College of Medicine, Gainesville

Bforlando@ufl.edu

The authors reported no potential conflict of interest relevant to this article.

doi: $10.12788 / \mathrm{fp} .0307$

\section{Functional medicine: Focusing on imbalances in core metabolic processes}

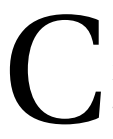
ould screening patients for cytokine markers help direct interventions to prevent quality-of-life deterioration? What evidence is there that a patient's methylenetetrahydrofolate reductase (MTHFR) genotype and baseline folate level can determine whether folate therapy will be needed to prevent stroke?

Favorable findings in these areas and others-eg, that specific probiotics benefit those with various gastrointestinal, respiratory, and lipid disorders-are strengthening support for the clinical approach of functional medicine (FM), which focuses on core functional processes: assimilation, defense and repair, energy, biotransformation and elimination, transport, communication, and structural integrity.

In this article, we describe the paradigm of FM, review its origins, and present the evidence base of selected topics: diagnostic testing, nutrition and supplements, probiotics, and chelation. As FM's popularity increases, a better understanding of it will help us educate our patients on this approach and implement some of its evidence-backed practices. In preparing this review we used keyword searches of PubMed, Embase, Web of Science, Cochrane Library, University of Florida Health Science Center Library eJournals, the Institute for Functional Medicine website, ${ }^{1}$ and Lifestyle Medicine textbook. ${ }^{2}$

\section{The core of functional medicine}

Complementary and alternative medicine (CAM) refers to medical practices diverging from standards of care and not generally taught at US medical schools or available at US hospitals. ${ }^{3}$ Integrative Medicine encompasses evidence-informed CAM, conventional Western medicine, and whole systems like FM. ${ }^{4}$

I FM aims to identify root causes of disease, emphasizing function as a dynamic process that can move back and forth on a continuum between health and disorder. ${ }^{5,6}$ There are 7 defining characteristics of FM: (1) patient centered vs disease centered; (2) systems biology approach acknowledging web-like interconnections of physiologic factors; (3) dynamic balance of gene-environment interactions; (4) personalized care based on biochemical individuality; (5) promotion of organ reserve and sustained health span; (6) health as a positive vitality - not merely the absence of disease; and (7) function vs pathology focused. ${ }^{5}$

I The concept of FM is not new. Its origins can be traced to the 19th century. Jeffrey Bland, PhD, credits the term's first use to Sir Willoughby F. Wade, MD, in an 1871 Lancet editorial, "Clinical lecture on functional medicine." Bland formulated the FM paradigm and in 1991 founded the Institute for Functional Medicine (the Institute), 
its main educational and certifying organization. ${ }^{7}$ The Institute certifies masters- or doctorate-prepared health professionals from both conventional and complementary health fields who complete 7 courses (Applying FM in Clinical Practice, Gastrointestinal, Environmental Health, Immune, Hormone, Cardiometabolic, and Bioenergetics), present 1 case report, and pass a written exam. In a retrospective cohort study, researchers found that the FM model was associated with greater improvements in patient-reported, health-related quality of life (QOL) compared with usual care. ${ }^{8}$

\section{Clinical model}

FM uses a comprehensive yet practical matrix for obtaining patient histories and for guiding diagnostic testing. ${ }^{9}$ Tools that support history-taking include a timeline; weeklong dietary survey; daily activity log; exercise, sleep and self-care questionnaires; and an environmental risk assessment. Instead of a review of symptoms arranged by organ system as is typical with conventional Western medicine, FM assesses the balance of core functional processes: assimilation, defense and repair, energy, biotransformation and elimination, transport, communication, and structural integrity.

Within the matrix, FM also recognizes 5 modifiable personal lifestyle factors: sleep and relaxation, exercise and movement, nutrition, stress, and relationships. When these lifestyle elements are influenced by specific predisposing factors (antecedents), discrete events precipitating illness (triggers), and ongoing physiologic processes (mediators), fundamental imbalances eventually result in the signs and symptoms characterizing diagnosable diseases.

The essence of the FM therapeutic plan is a discussion of lifestyle changes, personal strengths, and potential adherence challenges so the clinician can better offer assistive resources, which may include multidisciplinary referrals to personalized counselors such as a nutritionist, health coach, mind-body therapist, personal trainer, exercise physiologist, or physical therapist. While these professionals are not unique to FM, they are more fre- quently used by FM practitioners as part of the health care team.

\section{Diagnostic testing through a different lens}

A proposed solution to complex diseases recalcitrant to conventional modern treatments is a systems biology approach to health care.$^{10}$ Current laboratory testing such as liver enzymes, C-reactive protein (CRP), and leukocyte count provide some information on organ system homeostasis and molecular pathology, but even high-sensitivity CRP, which measures inflammation across the human system, is not adequate in cardiovascular disease (CVD) risk assessment. ${ }^{11}$ Genome-wide association studies suggest an association between biological pathways, genes, molecular markers, and QOL domains, and screening patients for cytokine markers may help provide prophylactic interventions to prevent QOL deterioration. ${ }^{12}$

With the purpose of being more systems-biology centric rather than disease centric, ${ }^{13}$ FM practitioners use tests to guide dietary recommendations and supplement selection, the evidence for which is found mostly in symptom-specific case series. ${ }^{14-16}$ Commonly used tests include MTHFR genotyping, comprehensive stool profiles, hormone and heavy metal panels, allergy panels, lactulose breath testing, micronutrient and advanced lipid panels, omega 3:6 ratios, and oxidative stress tests.

Some of these tests are performed by standard labs and are covered by insurance. Certain assays are primarily performed by specialized functional laboratories, such as comprehensive stool profiles, provoked heavy metal tests, non-IgE allergy panels, and oxidative stress tests. These laboratories also frequently offer direct-to-consumer orders. The latter situation raises a potential conflict of interest from supplement sales linked to laboratory testing and makes it imperative for health care professionals to understand the reliability and clinical utility of these tests in order to counsel patients accordingly.

The remainder of this article focuses on the evidence behind a subset of FM treatments, which typically include various
The essence of the functional medicine therapeutic plan is a discussion of lifestyle changes, personal strengths, and potential adherence challenges. 


\section{$>$}

Common FM tests are MTHFR genotyping, comprehensive stool profiles, hormone and heavy metal panels, allergy panels, lactulose breath testing, and micronutrient and advanced lipid panels. dietary interventions (elimination, cardiometabolic, detox) and multidomain lifestyle modifications. The practitioner's selection of dietary interventions, nutraceuticals (vitamins, minerals, essential fatty acids, botanicals), and probiotics is informed by results of different diagnostic tests.

\section{Nutrition and supplements}

Nearly one-third of older Americans are affected by at least 1 vitamin deficiency or anemia, and even those consuming an adequate diet have a substantial risk of any deficiency (16\%), although less so than those with an inadequate diet (57\%). ${ }^{17}$ The Western diet is known to be nutrient deficient, particularly in vitamin $\mathrm{D}$, thiamine, riboflavin, calcium, magnesium, and selenium. ${ }^{18}$ Dietary supplement nonusers have the highest risk of any deficiency $(40 \%)$ compared with users of "full-spectrum multivitamin-multimineral supplements" (14\%) and other dietary supplement users $(28 \%){ }^{17}$

Nevertheless, the US Preventive Services Task Force (USPSTF) concluded in 2014 that there are not enough data to make a recommendation for or against taking vitamins A, C, or $\mathrm{E}$; multivitamins with folic acid; or combinations of these vitamins for the primary prevention of CVD or cancer. ${ }^{19}$ USPSTF also does not recommend daily vitamin $\mathrm{D}$ and calcium supplementation in community-dwelling, postmenopausal women for primary prevention of fracture. ${ }^{20}$ Notwithstanding the lack of supplement recommendations for primary prevention, their benefits in patients with chronic disease is still being investigated. For example, a polyphenol-rich antioxidant may reduce cardiovascular complications in those with diabetes. $^{21}$

I Rethinking how nutrition studies are designed. Drawing on studies to determine the benefits of nutrition in chronic disease has been challenging. Factors that must be taken into account include the types of vitamin and mineral supplements patients use, nutrient absorption and utilization, and differing dietary assessment methods and reference values used. ${ }^{18}$ For example, vitamin and mineral absorption work best with whole food or fortified diets wherein specific nutri- ents are consumed together (eg, vitamin D and vitamin $\mathrm{E}$ with fat; non-heme iron with vitamin C). ${ }^{22-24}$ Foods with competing nutrients or "inhibitors" may even require absorption enhancers at minimum molar ratios. ${ }^{24}$

Adding to the complexity of vitamin and mineral absorption, botanical dietary supplements have their own modifying effects on micronutrient absorption..$^{25}$ These are just some of the reasons randomized controlled trials (RCTs) are fundamentally limited when investigating the health outcomes of diet and supplementation, and alternative study methods should be considered for future nutrition clinical trials to better inform clinicians who are prescribing supplements. ${ }^{26}$ In the meantime, nutrition plans can be individualized to patients' biological and cultural needs, ideally in conjunction with a multidisplinary team that includes dietitians, behaviorists, and exercise specialists. ${ }^{27}$

Nutrition is one of the most important environmental factors modulating genes and phenotypes (nutrigenomics), ${ }^{28,29}$ and recent studies on single nucleotide polymorphisms (SNPs) have reinforced the importance of considering the effect of genetic variation on dietary response (nutrigenetics). ${ }^{29,30}$ While nutrigenomics and nutrigenetics have the potential to improve the health of large populations by personalizing dietary advice based on genotype and phenotype, genetic variation occurring within a specific biochemical pathway makes generalizing genotype-based dietary advice to other populations complex.

For instance, Greenlandic Inuit, the indigenous people inhabiting the Arctic regions of Greenland, have a low incidence of CVD, largely due to "non-European" genetic variants that lower their LDL, protecting them from the oxidative stress of their diet high in polyunsaturated fats. ${ }^{31,32}$ In a study of European adults, phenotypic and phenotypic-plusgenotypic information did not enhance the effectiveness of the personalized nutrition advice, demonstrating that more research is needed in larger populations. ${ }^{33}$

Another specific, complex example of gene-nutrient interaction is the pathophysiologic outcome of polymorphisms in the MTHFR gene. ${ }^{30}$ MTHFR is a rate-limiting enzyme involved in folate and homocyste- 
ine metabolism, DNA and RNA biosynthesis, and DNA and protein methylation. MTHFR polymorphisms are common in otherwise healthy people, but some have been reported to increase chronic disease susceptibility via $M T H F R$ deficiency. The most common MTHFR variant is the SNP rs1801133 that reduces enzyme activity to $\sim 30 \%$ in homozygotes (677TT), which can lead to reduced folate bioavailability and mild-to-moderate hyperhomocysteinemia depending on one's dietary folate intake and food fortification.

Ine FM focus at variance with current recommendations. FM's approach to homocysteine and MTHFR genotyping for nutritional assessment, dietary counseling, and supplement advice contrasts with recommendations from meta-analyses ${ }^{34-36}$ and guidelines from the American Heart Association and USPSTF. ${ }^{37,38}$ While USPSTF does recommend all women planning or capable of pregnancy take 0.4 to $0.8 \mathrm{mg}$ of folic acid daily to prevent neural tube defects, ${ }^{39}$ MTHFR polymorphism screening to guide supplementation is not recommended during pregnancy given conflicting studies and uncertain clinical significance. ${ }^{40}$ Moreover, while it is generally agreed that the MTHFR C677T polymorphism, and in particular the homozygous MTHFR 677TT genotype, is an independent risk factor for hyperhomocysteinemia, neither variant genotype was an independent coronary artery disease risk factor in several large studies. ${ }^{41-43}$

However, study populations with generally high folate consumption or fortified foods may be a main confounding variable. ${ }^{44}$ For example, in a recent meta-analysis of an elderly population, the T-allele of the MTHFR C677T variant increased pooled stroke risk. While this increased stroke risk is highest in homozygotes, the association was statistically significant only in the Chinese cohort-a group that generally has poor dietary intake of folate and B12. ${ }^{45}$ Therefore, both MTHFR genotype and baseline folate level are important determinants of folate therapy efficacy in stroke prevention, and this may explain why US studies have not clearly identified a patient subset most likely to benefit. ${ }^{46} \mathrm{Fu}$ ture high-quality studies should measure baseline folate, target populations with moderate-to-severe hyperhomocysteinemia with specific MTHFR polymorphisms, and compare high- and low-dose B vitamins or use 5-methylfolate (the active form of folate).

\section{Probiotics}

Probiotics are used extensively in FM, and there are very few fields in conventional Western medicine where probiotics have not been researched. Interestingly, gut microbiota (microflora) change rapidly and individually to therapeutic dietary changes, both in composition (community) and function (metabolic plasticity), implicating gut microbiota as a mediator of dietary impact on host metabolism. ${ }^{47}$ This highlights the potential for tailored probiotics to transform dietary nonresponders (eg, those who do not routinely consume a high-fiber diet) into responders whose metabolism becomes enabled to counter such conditions as obesity and type 2 diabetes. ${ }^{48}$

Caveats with probiotic administration. While the strength of recommendation for probiotic therapy is increased by their safe use in pregnancy, infants, and immunocompromised populations, ${ }^{49-51}$ various harms (ranging from mild to severe) may be underreported ${ }^{52-54}$ In clinical practice, the effective probiotic strain(s), formulation, and dosage will vary not only by disease, but also from patient to patient, and may be dependent on nutritional factors such as vitamin $\mathrm{D},{ }^{55}$ diet, and even epigenetics. ${ }^{56}$

As we come to more fully recognize commensal microbiota as a major player in overall health with crosstalk in signaling pathways between intestinal bacterial and epithelial immune cells, future research is needed to help optimize probiotic dose, duration, route of administration, and whether microbial communities outperform singlespecies probiotics. ${ }^{50}$ Furthermore, much of a bacterial community's effect on the host is through its metabolites, and since certain species can be used interchangeably given similar metabolic activity and function, studies to understand how prebiotics and probiotics affect the host must analyze the metabolome, in addition to the bacterial community composition. ${ }^{51}$
Future research is needed to help optimize probiotic dose, duration, route of administration, and whether microbial communities outperform single-species probiotics. 


\section{$>$}

\section{Mechanistic}

microbiota

studies are

facilitating early

clinical

research in

numerous

diseases,

such as proof-ofconcept RCTs in atherosclerosis prevention.
I Alternative research methods could be informative. Similar to their limited evaluation of health outcomes in nutrition and supplement research, many RCTs examining the health benefits of probiotics often yield ambiguous results or fall short of valid conclusions because the underlying presuppositions are not met. ${ }^{26}$ For example, assuming the RCT uses a well-defined probiotic formulation, efficacy and generalizability can still be confounded by patient microbiota, diet, mucosa, immune system, and emotional status, which all affect probiotic activity/ potency. ${ }^{50}$ Under these circumstances, other research methods may be more suitable or supplemental-eg, Phase II trials, epidemiologic studies, single-case experiments (n-of-1 trials) and their meta-analyses, using historical controls, preclinical studies for conceptual and theoretical development, and clinical experience. ${ }^{26,57-59}$ Mechanistic microbiota studies are facilitating early clinical research in numerous diseases, ${ }^{60}$ such as proof-of-concept RCTs in atherosclerosis prevention $^{61}$ and brain function. ${ }^{62}$

It is currently unknown how nonvaginal microbiota affect the health of menopausal women; ${ }^{63,64}$ and while a multicenter RCT is underway to examine a novel probiotic's effect on menopausal symptoms and bone health, the supplemental study methods listed above could be employed as well.

Evidence of probiotic effectiveness. Positive evidence in RCTs and meta-analyses suggests that strain-specific probiotics are beneficial for infectious gastroenteritis, persistent antibiotic-associated diarrhea, infant colic, $^{50}$ irritable bowel syndrome (IBS), ${ }^{50,65}$ Clostridioides difficile prevention, ${ }^{66}$ inflammatory bowel disease (IBD), ${ }^{67}$ radiation-induced diarrhea, ${ }^{68,69}$ and nongastrointestinal conditions such as multiple sclerosis, ${ }^{70}$ upper respiratory infections, ${ }^{50}$ atopic dermatitis, ${ }^{50,71,72}$ surgical site infections, ${ }^{73,74}$ hyperlipidemia, ${ }^{50,75,76}$ and nonalcoholic steatohepatitis. ${ }^{77}$

However, because of knowledge gaps and low-quality evidence, the American Gastroenterological Association's (AGA) 2020 Clinical Practice Guidelines recommend "conditional” use of strain-specific probiotics only for necrotizing enterocolitis prevention (in pre-term, low-birth-weight infants), antibiotic-induced $C$ difficile prevention, and pouchitis. ${ }^{53,54}$ Additionally, the AGA's guidelines state that probiotics should only be used for $C$ difficile infection, IBD, or IBS in a clinical trial and not used at all for infectious gastroenteritis. ${ }^{53,54}$

\section{Chelation}

Chelation therapy is thought to inhibit metal-catalyzed oxidation reactions and inflammatory processes in tissues that lead to, and result from, accumulation of oxidative damage. While many FM practitioners recommend some form of chelation therapy, there is much controversy surrounding its use. Well-designed, large-scale clinical trials continue to emerge for what appears to be an old intervention with still uncertain clinical applications.

The first chelation agent, disodium ethylenediaminetetraacetic acid (EDTA), was synthesized in the early 1930s, but it was not until World War II that chelation therapy began with use of dimercaprol, a potent antidote for the arsenical chemical weapon lewisite. Disodium EDTA infusions were first used to treat angina pectoris in the mid-20th century, ${ }^{78}$ and use accelerated in the early 21 st century without a clear indication to treat CVD besides decades of anecdotes and case reports. ${ }^{79}$ Strong epidemiologic data support a causal association between heavy metals (arsenic, lead, cadmium, mercury, and copper) and $C V D,{ }^{80,81}$ but a distinct mechanism of action is not yet known.

Disodium EDTA was FDA approved in the 1950s for use in patients with hypercalcemia or ventricular arrhythmias from digitalis toxicity, but it was eventually withdrawn from the market in 2008, in part due to safety concerns following 3 deaths from severe hypocalcemia. ${ }^{82}$ These deaths were caused by inappropriate use related to rapid infusions, pediatric use, and in one case inadvertently administering disodium EDTA to a child instead of calcium EDTA (which can safely be bolused to treat pediatric lead toxicity). ${ }^{82-85}$

The Trial to Assess Chelation Therapy (TACT) was the first large, doubleblinded, placebo-controlled RCT to test post-myocardial infarction (MI) disodium 
EDTA infusions (weekly 3-hour infusions for 30 weeks followed by 10 infusions 2 to 8 weeks apart). ${ }^{86}$ Despite the chelation group having a modest reduction in the primary composite endpoint (hazard ratio $[\mathrm{HR}]=0.82$; 95\% CI, $0.69-0.99 ; \quad P=.035),{ }^{86}$ especially in those with diabetes ( $\mathrm{HR}=0.59 ; 95 \% \mathrm{CI}$, 0.44-0.79; $P<.001$; number needed to treat $[\mathrm{NNT}]=6.5),{ }^{87}$ the authors concluded these results were insufficient to support routine disodium EDTA chelation post MI. Moreover, the 2014 Guidelines for Chronic Ischemic Heart Disease only upgraded chelation therapy from "not recommended" to "uncertain." ${ }^{88}$

Nevertheless, to put into perspective the magnitude of chelation's treatment effect post MI in patients with diabetes, it can be compared to standard-of-care statin RCTs for secondary prevention, reported as follows. The absolute risk reduction (ARR) of coronary events with statin therapy in a high genetic risk group (the group with the greatest ARR) was $6.03 \%$ in the CARE trial and $6.87 \%$ in the PROVE IT-TIMI 22 trial, corresponding to a calculated NNT (1/ARR) of 16.6 and 14.6 respectively. ${ }^{89}$

In order to understand the hesitancy to accept chelation therapy as a reasonable CVD treatment, ${ }^{59}$ one must balance the modest effect demonstrated by TACT with its limitations, the greatest of which was the unusually large proportion of patients who withdrew their consent or were lost to followup $(\sim 18 \%) .{ }^{86}$ However, it is important to note that some patients withdrew after experiencing a primary endpoint and that at least $50 \%$ more were from the placebo group than the chelation group, ${ }^{86}$ rather than the reverse that was erroneously reported by Fihn et al. ${ }^{88}$ Unblinding was possible, but there were no significant differences in serious adverse events between groups.

Furthermore, since withdrawn subjects had similar CVD risk profiles, more withdrawals in the placebo arm increases the likelihood of more unrecorded primary outcome events in the placebo arm than treatment arm. One possibility is that these could have accentuated the benefit of chelation therapy, and that missing data reduced the reported efficacy. Because of TACT's profound findings for those with diabetes post MI, the TACT2
Phase 3 clinical trial underway through 2022 will further clarify chelation's utility for treating stable ischemic heart disease specifically in this group.

Interestingly, angiotensin-converting enzyme inhibitors, angiotensin receptor blockers, and aldose reductase inhibitors themselves inhibit advanced glycation end products (AGE) formation in diabetes, most likely via chelation. Increased protein glycation and accumulation of AGEs on tissue proteins during hyperglycemia (the Maillard reaction) are hypothesized to be fundamental in the pathogenesis of diabetic vascular complications. Consequently, chronic, lowdose chelation therapy may have a role in preventing and treating diabetic CVD and nephropathy. ${ }^{90}$

JFP

\section{ACKNOWLEDGEMENT}

The authors thank Ariel Pomputius, MLIS, for contributing to the literature searches during manuscript preparation.

\section{CORRESPONDENCE}

Frank A. Orlando, MD, UF Health Family Medicine Springhill, 4197 NW 86th Terrace; forlando@ufl.edu

\section{References}

1. The Institute for Functional Medicine. 2020. Accessed November 19, 2021. www.ifm.org/

2. Rippe JM, ed. Lifestyle Medicine. 3rd ed. CRC Press, Taylor \& Francis Group; 2019.

3. Eisenberg DM, Kessler RC, Foster C, et al. Unconventional medicine in the United States. Prevalence, costs, and patterns of use. NEngl J Med. 1993;328:246-252.

4. Ali A, Katz DL. Disease prevention and health promotion: how integrative medicine fits. Am J Prev Med. 2015;49(5 suppl 3): S230-S240.

5. Bland J. Defining function in the functional medicine model. Integr Med (Encinitas). 2017;16:22-25.

6. ABPS. Integrative medicine examination description. 2020 Accessed November 19, 2021. www.abpsus.org/integrativemedicine-description/

7. Bland JS. The natural roots of functional medicine. Integr Med (Encinitas). 2018;17:12-17.

8. Beidelschies M, Alejandro-Rodriguez M, Ji X, et al. Association of the functional medicine model of care with patient-reported health-related quality-of-life outcomes. JAMA Netw Open. 2019;2:e1914017.

9. The Institute for Functional Medicine. Functional medicine matrix: organizing clinical imbalances. 2020. Accessed November 19, 2021. www.ifm.org/news-insights/toolkit-functionalmedicine-matrix/

10. Schadt EE, Björkegren JL. NEW: Network-enabled wisdom in biology, medicine, and health care. Sci Transl Med. 2012;4:115rvl.

11. Curry SJ, Krist AH, Owens DK, et al. Risk assessment for cardiovascular disease with nontraditional risk factors: US Preventive Services Task Force recommendation statement. JAMA. 2018;320:272-280.

12. Sprangers MA, Thong MS, Bartels M, et al. Biological pathways, candidate genes, and molecular markers associated with qualityof-life domains: an update. Qual Life Res. 2014;23:1997-2013.

13. Bland J. Functional medicine: an operating system for integrative medicine. Integr Med (Encinitas). 2015;14:18-20.

14. Cutshall SM, Bergstrom LR, Kalish DJ. Evaluation of a functional
Chronic, lowdose chelation therapy may have a role in preventing and treating diabetic CVD and nephropathy. 
medicine approach to treating fatigue, stress, and digestive issues in women. Complement Ther Clin Pract. 2016;23:75-81.

15. Jaffe R. First line comprehensive care. Part II: Anthropogenic xenobiotics in functional medicine. Managing persisting bioaccumulating pollutants: toxic minerals, biocides, hormone mimics, solvents, and chemical disruptors. Semin Integr Med. 2005;3: 79-92.

16. Muran PJ, Muran SY, Beseler CL, et al. Breast health and reducing breast cancer risk: a functional medicine approach. J Altern Complement Med. 2015;21:321-326.

17. Bird JK, Murphy RA, Ciappio ED, et al. Risk of deficiency in multiple concurrent micronutrients in children and adults in the United States. Nutrients. 2017;9:655

18. ter Borg S, Verlaan S, Hemsworth J, et al. Micronutrient intakes and potential inadequacies of community-dwelling older adults: a systematic review. Br J Nutr. 2015;113:1195-1206.

19. Moyer VA, on behalf of the USPSTF. Vitamin, mineral, and multivitamin supplements for the primary prevention of cardiovascular disease and cancer: U.S. Preventive Services Task Force recommendation statement. Ann Intern Med. 2014;160:558-564.

20. Grossman DC, Curry SJ, Owens DK, et al. Vitamin D, calcium, or combined supplementation for the primary prevention of fractures in community-dwelling adults: U.S. Preventive Services Task Force recommendation statement. JAMA. 2018;319: 1592-1599.

21. Fenercioglu AK, Saler T, Genc E, et al. The effects of polyphenolcontaining antioxidants on oxidative stress and lipid peroxidation in Type 2 diabetes mellitus without complications. J Endocrinol Invest. 2010;33:118-124.

22. Silva MC, Furlanetto TW. Intestinal absorption of vitamin D: A systematic review. Nutr Rev. 2018;76:60-76.

23. Schmölz L, Birringer M, Lorkowski S, et al. Complexity of vitamin E metabolism. World J Biol Chem. 2016;7:14-43.

24. Teucher B, Olivares M, Cori H. Enhancers of iron absorption: ascorbic acid and other organic acids. Int J Vitam Nutr Res. 2004;74:403-419.

25. Gurley BJ, Tonsing-Carter A, Thomas SL, et al. Clinically relevant herb-micronutrient interactions: when botanicals, minerals, and vitamins collide. Adv Nutr. 2018;9:524s-532s.

26. Zeilstra D, Younes JA, Brummer RJ, et al. Perspective: fundamental limitations of the randomized controlled trial method in nutritional research: the example of probiotics. Adv Nutr. 2018;9: 561-571.

27. Kimokoti RW, Millen BE. Nutrition for the prevention of chronic diseases. Med Clin North Am. 2016;100:1185-1198.

28. Tucker KL, Smith CE, Lai CQ, et al. Quantifying diet for nutrigenomic studies. Annu Rev Nutr. 2013;33:349-371.

29. Fenech M, El-Sohemy A, Cahill L, et al. Nutrigenetics and nutrigenomics: viewpoints on the current status and applications in nutrition research and practice. $J$ Nutrigenet Nutrigenomics. 2011;4:69-89.

30. van Ommen B, van den Broek T, de Hoogh I, et al. Systems biology of personalized nutrition. Nutr Rev. 2017;75:579-599.

31. Fumagalli M, Moltke I, Grarup N, et al. Greenlandic Inuit show genetic signatures of diet and climate adaptation. Science. 2015;349:1343-1347.

32. Mathieson I, Lazaridis I, Rohland N, et al. Genome-wide patterns of selection in 230 ancient Eurasians. Nature. 2015;528:499-503.

33. Celis-Morales C, Livingstone KM, Marsaux CF, et al. Effect of personalized nutrition on health-related behaviour change: evidence from the Food4Me European randomized controlled trial. Int J Epidemiol. 2017;46:578-588.

34. Maron BA, Loscalzo J. The treatment of hyperhomocysteinemia. Annu Rev Med. 2009;60:39-54.

35. Li Y, Huang T, Zheng Y, et al. Folic acid supplementation and the risk of cardiovascular diseases: a meta-analysis of randomized controlled trials. J Am Heart Assoc. 2016;5:e003768.

36. Martí-Carvajal AJ, Solà I, Lathyris D, et al. Homocysteine-lowering interventions for preventing cardiovascular events. Cochrane Database Syst Rev. 2017;8:CD006612.

37. Goff DC, Jr., Lloyd-Jones DM, Bennett G, et al. 2013 ACC/AHA guideline on the assessment of cardiovascular risk: a report of the American College of Cardiology/American Heart Association Task Force on Practice Guidelines. Circulation. 2014;129 (25 suppl 2):S49-S73

38. USPSTF. Using nontraditional risk factors in coronary heart disease risk assessment: U.S. Preventive Services Task Force recommendation statement. Ann Intern Med. 2009;151:474-482.
39. Bibbins-Domingo K, Grossman DC, Curry SJ, et al. Folic acid supplementation for the prevention of neural tube defects: U.S. Preventive Services Task Force recommendation statement. JAMA. 2017;317:183-189.

40. Levin BL, Varga E. MTHFR: addressing genetic counseling dilemmas using evidence-based literature. J Genet Couns. 2016;25: 901-911.

41. Luo Z, Lu Z, Muhammad I, et al. Associations of the MTHFR rs 1801133 polymorphism with coronary artery disease and lipid levels: a systematic review and updated meta-analysis. Lipids Health Dis. 2018;17:191.

42. Clarke R, Bennett DA, Parish S, et al. Homocysteine and coronary heart disease: meta-analysis of MTHFR case-control studies, avoiding publication bias. PLoS Med. 2012;9:e1001177.

43. Kölling K, Ndrepepa G, Koch W, et al. Methylenetetrahydrofolate reductase gene C677T and A1298C polymorphisms, plasma homocysteine, folate, and vitamin B12 levels and the extent of coronary artery disease. Am J Cardiol. 2004;93:1201-1206.

44. Holmes MV, Newcombe P, Hubacek JA, et al. Effect modification by population dietary folate on the association between MTHFR genotype, homocysteine, and stroke risk: a meta-analysis of genetic studies and randomised trials. Lancet. 2011;378:584-594.

45. Chang G, Kuai Z, Wang J, et al. The association of MTHFR C677T variant with increased risk of ischemic stroke in the elderly population: a meta-analysis of observational studies. BMC Geriatr. 2019;19:331.

46. Huo Y, Li J, Qin X, et al. Efficacy of folic acid therapy in primary prevention of stroke among adults with hypertension in China: the CSPPT randomized clinical trial. JAMA. 2015;313:1325-1335.

47. Reid G. Probiotics: definition, scope and mechanisms of action. Best Pract Res Clin Gastroenterol. 2016;30:17-25.

48. Sonnenburg JL, Bäckhed F. Diet-microbiota interactions as moderators of human metabolism. Nature. 2016;535:56-64.

49. Van den Nieuwboer M, Brummer RJ, Guarner F, et al. The administration of probiotics and synbiotics in immune compromised adults: Is it safe? Benef Microbes. 2015;6:3-17.

50. Liu Y, Tran DQ, Rhoads JM. Probiotics in disease prevention and treatment. J Clin Pharmacol. 2018;58(suppl 10):S164-S179.

51. O'Connell TM. The application of metabolomics to probiotic and prebiotic interventions in human clinical studies. Metabolites. 2020;10:120.

52. Lerner A, Shoenfeld Y, Matthias T. Probiotics: if it does not help it does not do any harm. Really? Microorganisms. 2019;7:104.

53. Su G, Ko C, Bercik P, et al. AGA clinical practice guidelines on the role of probiotics in the management of gastrointestinal disorders. Gastroenterol. 2020;159:697-705.

54. Preidis GA, Weizman AV, Kashyap PC, et al. AGA technical review on the role of probiotics in the management of gastrointestinal disorders. Gastroenterology. 2020;159:708-738.e4.

55. Charoenngam N, Shirvani A, Kalajian TA, et al. The effect of various doses of oral vitamin $\mathrm{D}$ supplementation on gut microbiota in healthy adults: A randomized, double-blinded, dose-response study. Anticancer Res. 2020;40:551-556.

56. Cuevas-Sierra A, Ramos-Lopez O, Riezu-Boj JI, et al. Diet, gut microbiota, and obesity: links with host genetics and epigenetics and potential applications. Adv Nutr. 2019;10(suppl1):S17-S30.

57. APA. Clay RA. More than one way to measure. Monitor Psychol. 2010;4:52. Accessed November 19, 2021. www.apa.org/ monitor/2010/09/trials

58. Deaton A, Cartwright N. Understanding and misunderstanding randomized controlled trials. Soc Sci Med. 2018;210:2-21.

59. Bauchner H, Fontanarosa PB, Golub RM. Evaluation of the trial to assess chelation therapy (TACT): the scientific process, peer review, and editorial scrutiny. JAMA. 2013;309:1291-1292.

60. Durack J, Lynch SV. The gut microbiome: relationships with disease and opportunities for therapy. J Exp Med. 2019;216:20-40.

61. Matsumoto M, Kitada Y, Naito Y. Endothelial function is improved by inducing microbial polyamine production in the gut: a randomized placebo-controlled trial. Nutrients. 2019;11:1188.

62. Bagga D, Reichert JL, Koschutnig K, et al. Probiotics drive gut microbiome triggering emotional brain signatures. Gut Microbes. 2018;9:486-496.

63. Vieira AT, Castelo PM, Ribeiro DA, et al. Influence of oral and gut microbiota in the health of menopausal women. Front Microbiol. 2017;8:1884

64. Ribeiro AE, Monteiro NES, Moraes AVG, et al. Can the use of probiotics in association with isoflavone improve the symptoms of genitourinary syndrome of menopause? Results from a randomCONTINUED ON PAGE 498 


\section{CONTINUED FROM PAGE 488}

ized controlled trial. Menopause. 2018;26:643-652.

65. Hong YS, Hong KS, Park MH, et al. Metabonomic understanding of probiotic effects in humans with irritable bowel syndrome. J Clin Gastroenterol. 2011;45:415-425.

66. Shen NT, Maw A, Tmanova LL, et al. Timely use of probiotics in hospitalized adults prevents clostridium difficile infection: a systematic review with meta-regression analysis. Gastroenterol. 2017;152:1889-1900.e9.

67. Ganji-Arjenaki M, Rafieian-Kopaei M. Probiotics are a good choice in remission of inflammatory bowel diseases: a meta analysis and systematic review. J Cell Physiol. 2018;233:2091-2103.

68. Linn YH, Thu KK, Win NHH. Effect of probiotics for the prevention of acute radiation-induced diarrhoea among cervical cancer patients: a randomized double-blind placebo-controlled study. Probiotics Antimicrob Proteins. 2019;11:638-647.

69. Liu M-M, Li S-T, Shu Y, et al. Probiotics for prevention of radiation-induced diarrhea: a meta-analysis of randomized controlled trials. PLoS One. 2017;12:e178870.

70. Kouchaki E, Tamtaji OR, Salami M, et al. Clinical and metabolic response to probiotic supplementation in patients with multiple sclerosis: a randomized, double-blind, placebo-controlled trial. Clin Nutr. 2017;36:1245-1249.

71. Navarro-López V, Ramírez-Boscá A, Ramón-Vidal D, et al. Effect of oral administration of a mixture of probiotic strains on SCORAD index and use of topical steroids in young patients with moderate atopic dermatitis: a randomized clinical trial. JAMA Dermatol. 2018;154:37-43.

72. Wang HT, Anvari S, Anagnostou K. The role of probiotics in preventing allergic disease. Children (Basel). 2019;6:24.

73. Kasatpibal N, Whitney JD, Saokaew S, et al. Effectiveness of probiotic, prebiotic, and synbiotic therapies in reducing postoperative complications: a systematic review and network meta-analysis. Clin Infect Dis. 2017;64(suppl2):S153-S160.

74. Liu PC, Yan YK, Ma YJ, et al. Probiotics reduce postoperative infections in patients undergoing colorectal surgery: a systematic review and meta-analysis. Gastroenterol Res Pract. 2017;2017:6029075

75. Hendijani E, Akbari V. Probiotic supplementation for management of cardiovascular risk factors in adults with type II diabetes: a systematic review and meta-analysis. Clin Nutr. 2018;37:532-541

76. Wu Y, Zhang Q, Ren Y, et al. Effect of probiotic Lactobacillus on lipid profile: a systematic review and meta-analysis of randomized, controlled trials. PLoS One. 2017;12:e0178868.

77. Ferolla SM, Couto CA, Costa-Silva L, et al. Beneficial effect of synbiotic supplementation on hepatic steatosis and anthropometric parameters, but not on gut permeability in a population with nonalcoholic steatohepatitis. Nutrients. 2016;8:397.
78. Clarke CN, Clarke NE, Mosher RE. Treatment of angina pectoris with disodium ethylene diamine tetraacetic acid. Am J Med Sci. 1956;232:654-666.

79. Barnes PM, Bloom B, Nahin RL. Complementary and alternative medicine use among adults and children: United States, 2007. Natl Health Stat Report. 2008:1-23.

80. Chowdhury R, Ramond A, O'Keeffe LM, et al. Environmental toxic metal contaminants and risk of cardiovascular disease: systematic review and meta-analysis. BMJ. 2018;362:k3310.

81. Zhuang X, Ni A, Liao L, et al. Environment-wide association study to identify novel factors associated with peripheral arterial disease: evidence from the National Health and Nutrition Examination Survey (1999-2004). Atherosclerosis. 2018;269:172-177.

82. Wax PM. Current use of chelation in American health care. J Med Toxicol. 2013:9;303-307.

83. CDC. Deaths associated with hypocalcemia from chelation therapy-Texas, Pennsylvania, and Oregon, 2003-2005. MMWR Morb Mortal Wkly Rep. 2006;55:204-207.

84. Atwood KC, Woeckner E. In pediatric fatality, edetate disodium was no accident. Clin Toxicol (Phila). 2009;47:256.

85. Baxter AJ, Krenzelok EP. Pediatric fatality secondary to EDTA chelation. Clin Toxicol (Phila). 2008;46:1083-1084.

86. Lamas GA, Goertz C, Boineau R, et al. Effect of disodium EDTA chelation regimen on cardiovascular events in patients with previous myocardial infarction: the TACT randomized trial. JAMA. 2013;309:1241-1250.

87. Escolar E, Lamas GA, Mark DB, et al. The effect of an EDTA-based chelation regimen on patients with diabetes mellitus and prior myocardial infarction in the Trial to Assess Chelation Therapy (TACT). Circ Cardiovasc Qual Outcomes. 2014;7:15-24.

88. Fihn SD, Blankenship JC, Alexander KP, et al. 2014 ACC/AHA/ AATS/PCNA/SCAI/STS focused update of the guideline for the diagnosis and management of patients with stable ischemic heart disease: a report of the American College of Cardiology/American Heart Association Task Force on Practice Guidelines, and the American Association for Thoracic Surgery, Preventive Cardiovascular Nurses Association, Society for Cardiovascular Angiography and Interventions, and Society of Thoracic Surgeons. J Am Coll Cardiol. 2014;64:1929-1949.

89. Mega JL, Stitziel NO, Smith JG, et al. Genetic risk, coronary heart disease events, and the clinical benefit of statin therapy: an analysis of primary and secondary prevention trials. Lancet 2015;385:2264-2271.

90. Nagai R, Murray DB, Metz TO, et al. Chelation: a fundamental mechanism of action of AGE inhibitors, AGE breakers, and other inhibitors of diabetes complications. Diabetes. 2012;61: 549-559. 